\title{
RENAL INVOLVEMENT IN DERMATOMYOSITIS: CASE REPORT
}

Alisson Regis ${ }^{1, \star}$, Gustavo Behrens ${ }^{1}$, Washington dos Santos², Thaiza Gonçalves ${ }^{3}$, Mittermayer Santiago ${ }^{1}$

1.Universidade Federal da Bahia, Salvador (BA), Brazil. 2.Fundação Oswaldo Cruz, Salvador (BA), Brazil. 3.Unidade de Saúde Bráulio Santos Andrade, Itatim (BA), Brazil.

*Corresponding author: alisson_ios@hotmail.com

\section{BACKGROUND}

Dermatomyositis (DM) is an idiopathic inflammatory myopathy characterized by inflammation of the skeletal musculature and typical skin involvement. Renal involvement in DM is rare, described in isolated case reports. The authors present a case of a patient diagnosed with DM who developed nephrotic proteinuria.

\section{CASE REPORT}

A 48-year-old black female started proximal muscle weakness, heliotrope, Gottron's sign and papules 19 years ago. Laboratory tests showed increased muscle enzymes (CPK $717 \mathrm{mg} / \mathrm{dL}$ ), being diagnosed with DM. During follow-up she developed calcinosis. Initially, she used prednisone and dapsone, but recently she was on hydroxychloroquine $400 \mathrm{mg} /$ day and azathioprine $100 \mathrm{mg} /$ day. Due to stability, hydroxychloroquine was suspended in February 2020. In May 2020, she developed lower limb edema. Laboratory tests revealed urinalysis with proteinuria $1+$ to $4+; 24$-h proteinuria of $4,914 \mathrm{~g}$; albumin $2.5 \mathrm{~g} / \mathrm{dL}$; serum creatinine $0.5 \mathrm{mg} / \mathrm{dL}$, antinuclear antibody (ANA) was positive 1:80 quasi-homogeneous pattern, but other autoantibodies were negative (anti-Sm, anti-DNA, anti-RNP, anti-Jo1) and complement fractions (C3 and $\mathrm{C} 4$ ) were normal. A renal biopsy revealed normal looking glomeruli (1 sclerosed). Chronic tubulointerstitial changes extended for less than $5 \%$ of the represented cortical. Immunofluorescence revealed only traces of IgA in mesangium with $\lg$ M, IgG, kappa chain, C3, C1q and fibrinogen negative. The final histological diagnosis was minimal change disease (Fig. 1). A panoramic image of the renal cortex showing normal glomeruli (H\&E). Tubules are normal or have protein cylinders. B-detail of a glomerulus without changes (PAS). C and D immunofluorescence findings: IgA staining in mesangium and IgG (negative). Prednisone (1 mg/kg/day) was started, azathioprine (2 mg/kg/day) was maintained, and hydroxychloroquine was resumed ( $5 \mathrm{mg} / \mathrm{kg} /$ day) with partial improvement of proteinuria.

\section{CONCLUSION}

Most cases of DM involve skin and muscles; however, some extramuscular manifestations are relatively common. Data on renal involvement in DM remain scarce in the literature. Renal involvement in DM is divided in two distinct pathophysiological mechanisms, one associated with acute kidney injury due to rhabdomyolysis and another as an immunomediated glomerulonephritis, which can occur simultaneously with myopathy, or even many years after the diagnosis, as in the present case. The histopathological patterns are diffuse proliferative glomerulonephritis, mesangial proliferative glomerulonephritis, IgA nephropathy, focal and segmental glomerulosclerosis, membranous nephropathy and minimal change disease.
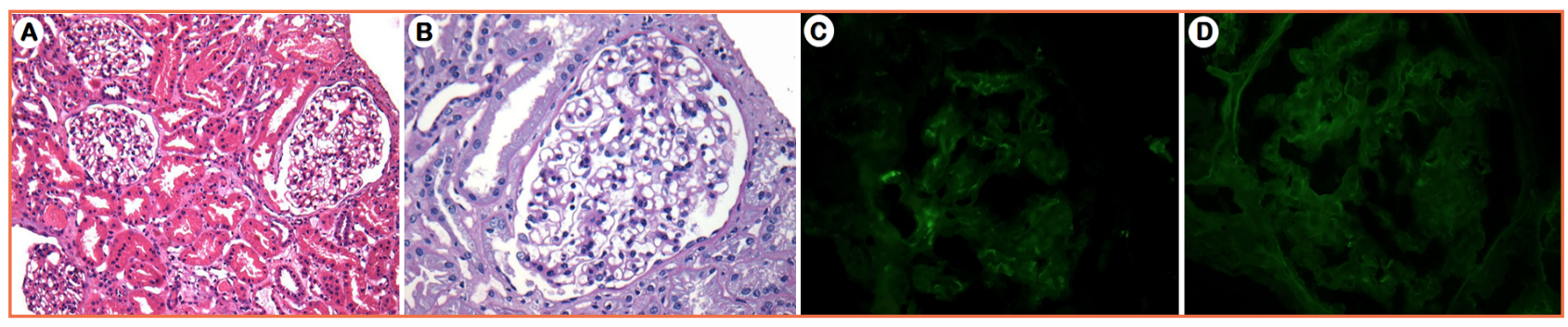

Figure 1. Final histological diagnosis. 